CONFORMAL GEOMETRY AND DYNAMICS

An Electronic Journal of the American Mathematical Society

Volume 11, Pages 1-11 (January 10, 2007)

S $1088-4173(07) 00148-8$

\title{
A NOTE ON CONFORMAL CONNECTIONS ON LIGHTLIKE HYPERSURFACES
}

\author{
CYRIAQUE ATINDOGBE AND LIONEL BERARD-BERGERY
}

\begin{abstract}
Degenerate submanifolds of pseudo-Riemannian manifolds are quite difficult to study because there is no preferred connection when the submanifold is not totally geodesic. For the particular case of degenerate totally umbilical hypersurfaces, we show that there are "Weyl" connections adapted to the induced structure on the hypersurface. We begin the study of these with their holonomy.
\end{abstract}

\section{INTRODUCTION}

If $(\bar{M}, \bar{g})$ is a Riemannian manifold, any submanifold $M$ of $\bar{M}$ has an induced Riemannian metric $g$. Moreover, the Levi-Civita connection $D$ of the metric $g$ is nothing but the orthogonal projection along $M$ of the Levi-Civita connection $\bar{D}$ of $\bar{g}$. This is the starting point of the theory of Riemannian submanifolds, and it goes back to Gauss.

If $(\bar{M}, \bar{g})$ is a pseudo-Riemannian manifold, the above procedure does not apply as well when the induced "metric" $g$ on $M$ is degenerate. First, there is no longer an orthogonal projection of $T \bar{M}$ onto $T M$ along $M$. Furthermore, there is no "Levi-Civita" connection for $g$ in the general case.

In this paper, we will consider the special case of degenerate hypersurfaces. It is known that $(M, g)$ admits a torsion-free connection $\nabla$ with $g$-parallel $(\nabla g=0)$ if and only if $(M, g)$ is totally geodesic in $(\bar{M}, \bar{g})$. When $(M, g)$ is not totally geodesic, one may still choose a torsion-free connection $\nabla$ on $M$ by projecting $\bar{D}$, but there are some choices for such a projection. Such "projected" connections are studied for example in [5], with the choice of an auxiliary screen distribution.

In this paper we will consider only the special case of totally umbilical hypersurfaces. We remark that a projected connection (as above) cannot be a "Weyl" connection for $(M, g)$. We give a precise definition of a Weyl connection in $\$ 3$ (Definition 3.3), but this is the same as the classical definition for nondegenerate metrics.

We illustrate that fact in $\$ 4$ with the basic example of the light cone at the origin inside the flat 3-dimensional Lorentz space.

Received by the editors December 1, 2005.

2000 Mathematics Subject Classification. Primary 53C50, 53C05, 53C29.

Key words and phrases. Lightlike hypersurface, screen distribution, conformal connection, holonomy group.

The first author thanks the Agence Universitaire de la Francophonie (AUF) for support with a one year research grant, along with the Institut Elie Cartan (IECN, UHP-Nancy I) for research facilities during the completion of this work.

(C)2007 American Mathematical Society Reverts to public domain 28 years from publication 
On the other hand, we show in $\$ 5$ that there are Weyl connections for any totally umbilical $(M, g)$. We hope that these Weyl connections will be more suited than projected connections for the study of totally umbilical degenerate hypersurfaces. In $₫ 6$, we illustrate that point of view with a preliminary study of the holonomy of these Weyl connections.

\section{Preliminaries on LightLike hyPersurfaces}

Let $M$ be a hypersurface of an $(n+2)$-dimensional pseudo-Riemannian manifold $(\bar{M}, \bar{g})$ of index $0<\nu<n+2$. In the classical theory of nondegenerate hypersurfaces, the normal bundle has trivial intersection $\{0\}$ with the tangent one and plays an important role in the introduction of main geometric objects. In the case of lightlike (degenerate, null) hypersurfaces, the situation is totally different. The normal bundle $T M^{\perp}$ is a rank-one distribution over $M: T M^{\perp} \subset T M$ and then coincides with the so-called radical distribution $\operatorname{RadTM}=T M \cap T M^{\perp}$. Hence, the induced metric tensor field $g$ is degenerate and has rank $n$. The following characterisation is proved in [5].

Proposition 2.1. Let $(M, g)$ be a hypersurface of an $(n+2)$-dimensional pseudoRiemannian manifold $(\bar{M}, \bar{g})$. Then the following assertions are equivalent.

(i) $M$ is a lightlike hypersurface of $\bar{M}$.

(ii) $g$ has constant rank $n$ on $M$.

(iii) $T M^{\perp}=\bigcup_{x \in M} T_{x} M^{\perp}$ is a distribution on $M$.

A complementary bundle of $T M^{\perp}$ in $T M$ is a rank $n$ nondegenerate distribution over $M$. It is called a screen distribution on $M$ and is often denoted by $S(T M)$. A lightlike hypersurface endowed with a specific screen distribution is denoted by the triple $(M, g, S(T M))$. As $T M^{\perp}$ lies in the tangent bundle, the following result has an important role in studying the geometry of a lightlike hypersurface.

Proposition $2.2([5])$. Let $(M, g, S(T M))$ be a lightlike hypersurface of $(\bar{M}, \bar{g})$ with a given screen distribution $S(T M)$. Then there exists a unique rank 1 vector subbundle $\operatorname{tr}(T M)$ of $\left.T \bar{M}\right|_{M}$, such that for any nonzero section $\xi$ of $T M^{\perp}$ on a coordinate neighbourhood $\mathcal{U} \subset M$, there exists a unique section $N$ of $\operatorname{tr}(T M)$ on $\mathcal{U}$ satisfying

$$
\bar{g}(N, \xi)=1
$$

and

$$
\bar{g}(N, N)=\bar{g}(N, W)=0, \quad \forall W \in \Gamma\left(\left.S T\right|_{\mathcal{U}}\right)
$$

Here and in the sequel we denote by $\Gamma(E)$ the $\mathcal{F}(M)$-module of smooth sections of a vector bundle $E$ over $M, \mathcal{F}(M)$ being the algebra of smooth functions on $M$. Also, by $\perp$ and $\oplus$ we denote the orthogonal and nonorthogonal direct sum of two vector bundles. By Proposition 2.2 we may write down the following decompositions:

$$
\begin{gathered}
T M=S(T M) \perp T M^{\perp}, \\
\left.T \bar{M}\right|_{M}=T M \oplus \operatorname{tr}(T M),
\end{gathered}
$$

and

$$
\left.T \bar{M}\right|_{M}=S(T M) \perp\left(T M^{\perp} \oplus \operatorname{tr}(T M)\right) .
$$


As it is well known, we have the following.

Definition 2.3. Let $(M, g, S(T M))$ be a lightlike hypersurface of $(\bar{M}, \bar{g})$ with a given screen distribution $S(T M)$. The induced connection, say $\nabla$, on $M$ is defined by

$$
\nabla_{X} Y=Q\left(\bar{\nabla}_{X} Y\right)
$$

where $\bar{\nabla}$ denotes the Levi-Civita connection on $(\bar{M}, \bar{g})$ and $Q$ is the projection on $T M$ with respect to the decomposition (2.4).

Remark 2.4. Notice that the induced connection $\nabla$ on $M$ depends on both $g$ and the specific given screen distribution $S(T M)$ on $M$.

By respective projections $Q$ and $I-Q$, we have Gauss and Weingarten formulae in the form

$$
\begin{gathered}
\bar{\nabla}_{X} Y=\nabla_{X} Y+h(X, Y) \quad \forall X, Y \in \Gamma(T M), \\
\bar{\nabla}_{X} V=-A_{V} X+\nabla_{X}^{t} V \quad \forall X \in \Gamma(T M), \quad \forall V \in \Gamma(\operatorname{tr}(T M)) .
\end{gathered}
$$

Here, $\nabla_{X} Y$ and $A_{V} X$ belong to $\Gamma(T M)$. Hence

- $h$ is a $\Gamma(\operatorname{tr}(T M))$-valued symmetric $\mathcal{F}(M)$-bilinear form on $\Gamma(T M)$,

- $A_{V}$ is an $\mathcal{F}(M)$-linear operator on $\Gamma(T M)$, and

- $\nabla^{t}$ is a linear connection on the lightlike transversal vector bundle $\operatorname{tr}(T M)$.

Let $P$ denote the projection morphism of $\Gamma(T M)$ on $\Gamma(S(T M))$ with respect to the decomposition (2.3). We have

$$
\begin{gathered}
\nabla_{X} P Y=\stackrel{\star}{\nabla}_{X} P Y+h^{*}(X, P Y) \quad \forall X, Y \in \Gamma(T M), \\
\nabla_{X} U=-\stackrel{\star}{A}_{U} X+\nabla_{X}^{* t} U \quad \forall X \in \Gamma(T M), \quad \forall U \in \Gamma\left(T M^{\perp}\right) .
\end{gathered}
$$

Here $\stackrel{\star}{\nabla}_{X} P Y$ and $\stackrel{\star}{A}_{U} X$ belong to $\Gamma(S(T M)), \stackrel{\star}{\nabla}$ and $\nabla^{* t}$ are linear connections on $S(T M)$ and $T M^{\perp}$, respectively. Hence

- $h^{*}$ is a $\Gamma\left(T M^{\perp}\right)$-valued $\mathcal{F}(M)$-bilinear form on $\Gamma(T M) \times \Gamma(S(T M))$, and

- $\stackrel{\star}{A} U_{U}$ is a $\Gamma(S(T M))$-valued $\mathcal{F}(M)$-linear operator on $\Gamma(T M)$.

They are the second fundamental form and the shape operator of the screen distribution, respectively.

Equivalently, consider a normalizing pair $\{\xi, N\}$ as in the Theorem 2.2, Then, (2.7) and (2.8) take the form

$$
\bar{\nabla}_{X} Y=\nabla_{X} Y+B(X, Y) N \quad \forall X, Y \in \Gamma\left(\left.T M\right|_{\mathcal{U}}\right),
$$

and

$$
\bar{\nabla}_{X} N=-A_{N} X+\tau(X) N \quad \forall X \in \Gamma\left(\left.T M\right|_{\mathcal{U}}\right),
$$

where we put locally on $\mathcal{U}$,

$$
\begin{gathered}
B(X, Y)=\bar{g}(h(X, Y), \xi), \\
\tau(X)=\bar{g}\left(\nabla_{X}^{t} N, \xi\right) .
\end{gathered}
$$

It is important to stress the fact that the local second fundamental form $B$ in (2.13) does not depend on the choice of the screen distribution. 
We also define (locally) on $\mathcal{U}$ the following:

$$
\begin{gathered}
C(X, P Y)=\bar{g}\left(h^{*}(X, P Y), N\right), \\
\varphi(X)=-\bar{g}\left(\nabla_{X}^{\star t} \xi, N\right) .
\end{gathered}
$$

Thus, one has for $X \in \Gamma(T M)$,

$$
\begin{gathered}
\nabla_{X} P Y=\stackrel{\star}{\nabla} X P Y+C(X, P Y) \xi, \\
\nabla_{X} \xi=-\stackrel{\star}{A} \xi_{\xi} X+\varphi(X) \xi .
\end{gathered}
$$

It is straightforward to verify that for $X, Y \in \Gamma(T M)$,

$$
\begin{gathered}
B(X, \xi)=0, \\
B(X, Y)=g\left({ }^{\star}{ }_{\xi} X, Y\right), \\
\stackrel{\star}{A}_{\xi} \xi=0 .
\end{gathered}
$$

The linear connection $\nabla^{\star}$ from $(2.9)$ is a metric connection on $S(T M)$ and we have for all tangent vector fields $X, Y$ and $Z$ in $T M$,

$$
\left(\nabla_{X} g\right)(Y, Z)=B(X, Y) \eta(Z)+B(X, Z) \eta(Y)
$$

$$
\eta(\cdot)=\bar{g}(N, \cdot) .
$$

The induced connection $\nabla$ is torsion-free, but not necessarily $g$-metric. Also, on the geodesibility of $M$ the following is known.

Theorem 2.5 ([5, p. 88]). Let $(M, g, S(T M))$ be a lightlike hypersurface of a pseudo-Riemannian manifold $(\bar{M}, \bar{g})$. Then the following assertions are equivalent:

(i) $M$ is totally geodesic.

(ii) $h$ (or equivalently B) vanishes identically on $M$.

(iii) $\stackrel{\star}{A}_{U}$ vanishes identically on $M$, for any $U \in \Gamma\left(T M^{\perp}\right)$.

(iv) The connection $\nabla$ induced by $\bar{\nabla}$ on $M$ is torsion-free and metric.

(v) $T M^{\perp}$ is a parallel distribution with respect to $\nabla$.

(vi) $T M^{\perp}$ is a Killing distribution on $M$.

It turns out that if $(M, g)$ is not totally geodesic, there is no connection that is, at the same time, torsion-free and $g$-metric. But there is no unicity of such a connection in case there is any.

\section{Some facts about total umbilicity}

Definition 3.1. A lightlike hypersurface $(M, g)$ is totally umbilical if its second fundamental form $B$ is proportional to the induced metric $g$ pointwise, i.e. on each coordinate neighbourhood $\mathcal{U} \subset M$, there exists a smooth function $\lambda$ such that

$$
B(X, Y)=\lambda g(X, Y), \quad \forall X, Y \in \Gamma\left(\left.T M\right|_{\mathcal{U}}\right),
$$

or equivalently,

$$
\stackrel{\star}{A_{\xi}} P X=\lambda P X, \quad \forall X \in \Gamma\left(\left.T M\right|_{\mathcal{U}}\right) .
$$

If the function $\lambda$ is nowhere vanishing on $M$, then the latter is said to be proper totally umbilic. 
It is an easy matter to verify that this is an intrinsic notion that is independent on $\mathcal{U}$, the choice of a screen distribution, $\xi$ (and hence $N$ as in Proposition 2.2).

In some sense, total umbilicity is the nearest situation from being totally geodesic ( $\lambda$ is identically zero). Also, relation (3.2) may be written for a given $\xi$ in $\operatorname{Rad}(T M)$ as

$$
\bar{g}\left(\bar{\nabla}_{X} Y, \xi\right)=\alpha(\xi) g(X, Y)
$$

with $\alpha$ a 1 -form on $\operatorname{Rad}(T M)$ and $\alpha(\xi)=\lambda$. Hence, $(X, Y) \mapsto \bar{g}\left(\bar{\nabla}_{X} \xi, Y\right)=$ $-\alpha(\xi) g(X, Y)$ is a bilinear symmetric form on $\Gamma(T M)$.

Proposition 3.2. A lightlike hypersurface $(M, g)$ is totally umbilic if and only if there exists a 1-form $\alpha$ on RadTM such that, for any section $\xi$ of RadTM,

$$
\mathcal{L}_{\xi} g=-2 \alpha(\xi) g \text {. }
$$

Furthermore, $(M, g)$ is proper totally umbilic if and only if $\alpha$ is everywhere nonzero.

Proof. By using (2.18) and (2.20) we have

$$
\mathcal{L}_{\xi} g(X, Y)=-2 B(X, Y)
$$

for all tangent vector fields $X$ and $Y$ in $\Gamma(T M)$. Then, the equivalence follows (3.1).

In a (pseudo-) Riemannian setting, manifolds $M^{n}$ with conformal structure $[g]$ and torsion-free connection $D$, such that parallel translation induces conformal transformations, are called Weyl manifolds. In this respect we give the following.

Definition 3.3. A connection $\nabla$ on a lightlike hypersurface $(M, g)$ is said to be conformal if the covariant derivative of $g$ is proportional to $g$ in the following precise sense that there exists a 1 -form $\theta$ such that the following,

$$
\nabla g=\theta \otimes g,
$$

holds. If in addition, $\nabla$ is torsion-free, it is said to be a Weyl connection.

We were tempted to hope from Proposition 3.2, the induced metric $g$ failing to be parallel with respect to the induced connection, that the latter should be conformal. Unfortunately, it is not the case, as is shown in the following.

Theorem 3.4. Let $(M, g, S(T M))$ be a lightlike hypersurface with a given screen distribution $S(T M)$. Then, the induced connection is a Weyl connection if and only if $(M, g)$ is totally geodesic.

In particular, the induced connection on a proper totally umbilical lightlike hypersurface $(M, g, S(T M))$ is never a Weyl connection.

Proof. Assume that the induced connection $\nabla$ satisfies (3.6) for some smooth 1-form $\theta$ defined on $M$. Then, from (2.22) we get

for all vector fields $X, Y$ and $Z$ in $\Gamma(T M)$. In (3.7), for $Y=\xi \in R a d T M$, using (2.19) leads to $B(X, Z)=0$ for all vector fields $X$ and $Z$ in $\Gamma(T M)$, which is equivalent to saying that $(M, g)$ is totally geodesic. The last assertion is immediate since proper totally umbilic that leads to $B$ is everywhere nonzero.

We shall show that, indeed, there always exists Weyl connections on proper totally umbilic lightlike hypersurfaces. We begin with an elementary example. 


\section{An elementary EXAmple}

Consider the Lorentz space $\mathbb{R}_{1}^{3}$ that is $\mathbb{R}^{3}$ with the flat Lorentz structure associated with the quadratic form

$$
\bar{q}(u)=x^{2}+y^{2}-z^{2}
$$

for all $u \in \mathbb{R}^{3}$.

Let $\bigwedge_{0}^{2}$ be the light cone at the origin,

$$
\bigwedge_{0}^{2}=\left\{u \in \mathbb{R}^{3} \mid u \neq 0 \text { and } \bar{q}(u)=0\right\} .
$$

Consider a cylindric coordinates system $(r, \theta, z)$ on $\mathbb{R}^{3}$ and the associated frame field $\left(\partial_{r}, \partial_{\theta}, \partial_{z}\right)$ with

$$
\left\{\begin{array}{l}
\partial_{r}=\cos \theta \partial_{x}+\sin \theta \partial_{y} \\
\partial_{\theta}=-\frac{1}{r} \sin \theta \partial_{x}+\frac{1}{r} \cos \theta \partial_{y} \\
\partial_{z}=\partial_{z} .
\end{array}\right.
$$

Then, it is easy to show that $\xi=\partial_{r}+\partial_{z}$ and $\partial_{\theta}$ are in $T \bigwedge_{0}^{2}$ and that

$$
\langle\xi, \xi\rangle=\left\langle\xi, \partial_{\theta}\right\rangle=0
$$

where $\bar{g}=\langle$,$\rangle denotes the Lorentz metric on \mathbb{R}_{1}^{3}$. So, $\left\{\xi, \partial_{\theta}\right\}$ is a frame field on $\bigwedge_{0}^{2}$ and we have $\operatorname{RadT} \bigwedge_{0}^{2}=\operatorname{span}\left\{\xi=\partial_{r}+\partial_{z}\right\}$. Thus, $\bar{B}=\left\{\xi, \partial_{\theta}, \partial_{z}\right\}$ is a frame field on $\mathbb{R}_{1}^{3} \backslash\{r=0\}$ (which contains $\bigwedge_{0}^{2}$ ), with $T \bigwedge_{0}^{2}=\operatorname{span}\left\{\xi, \partial_{\theta}\right\}$. With respect to $\bar{B}$, we have

$$
\bar{g}_{\mid \bar{B}}=\left(\begin{array}{ccc}
0 & 0 & -1 \\
0 & \frac{1}{r^{2}} & 0 \\
-1 & 0 & -1
\end{array}\right) .
$$

Let $\bar{D}$ denote the Levi-Civita connection on $\mathbb{R}_{1}^{3}$. Then, we have

$$
\left\{\begin{array}{l}
\bar{D}_{\xi} \xi=0, \\
\bar{D}_{\xi} \partial_{\theta}=-\frac{1}{r} \partial_{\theta}, \\
\bar{D}_{\partial_{\theta} \xi}=-\frac{1}{r} \partial_{\theta}, \\
\bar{D}_{\partial_{\theta} \partial_{\theta}}=-\frac{1}{r^{3}} \partial_{z} .
\end{array}\right.
$$


Thus, $\bigwedge_{0}^{2}$ is proper totally umbilical with $\lambda=\frac{1}{r}$. Now, define on $\bigwedge_{0}^{2}$ a connection $\stackrel{0}{\nabla}$ as follows:

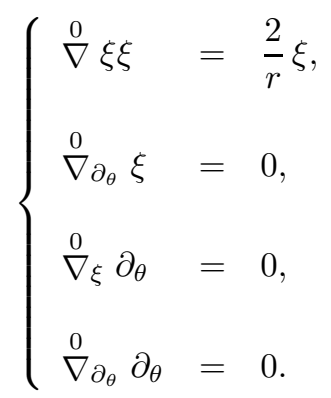

Let $g$ denote the induced metric on $\Lambda_{0}^{2}$. Then, by straightforward computation using (4.6) and (4.4), one gets for all tangent vector fields $X$ and $Y$ in $T \wedge_{0}^{2}$,

$$
(\stackrel{0}{\nabla} \times g)(\xi, Y)=0
$$

and

$$
\left(\stackrel{0}{\nabla}_{X} g\right)\left(\partial_{\theta}, \partial_{\theta}\right)=-2 \frac{X \cdot(r)}{r} \cdot \frac{1}{r^{2}} .
$$

Combining (4.7), (4.8) and (4.4), we deduce that

$$
\stackrel{0}{\nabla} g=\omega \otimes g
$$

with

$$
\omega=\frac{2}{r} \bar{g}\left(\cdot, \partial_{z}\right)
$$

Thus, since $\stackrel{0}{\nabla}$ is torsion-free by construction, it is a Weyl connection on $\bigwedge_{0}^{2}$.

From above enumerated facts, one can wonder whether the fact of admitting a Weyl connection characterizes completely proper totally umbilical lightlike hypersurfaces. We are going to give a positive answer to this question.

\section{A total Umbilicity CRITERion}

Theorem 5.1. For an $(M, g)$ lightlike hypersurface of a pseudo-Riemannian manifold $(\bar{M}, \bar{g})$ to be totally umbilical, it is necessary and sufficient that it admits a Weyl connection.

Proof. The condition is sufficient. Indeed, suppose there exists a Weyl connection $\nabla$ on $(M, g)$, that is, $\nabla$ is torsion-free and there exists on $M$ a smooth 1 -form $\theta$ such that $\nabla g=\theta \otimes g$. Then, applying this relation to $\xi$, and arbitrary $X$ and $Y$ in $T M$, we get

$$
\xi \cdot g(X, Y)-g\left(\nabla_{\xi} X, Y\right)-g\left(X, \nabla_{\xi} Y\right)=\theta(\xi) g(X, Y)
$$

or equivalently, since $\nabla$ is torsion-free,

$$
\left(\mathcal{L}_{\xi} g\right)(X, Y)=\theta(\xi) g(X, Y)+g\left(\nabla_{X} \xi, Y\right)+g\left(\nabla_{Y} \xi, X\right) .
$$

Now, we write $\nabla g=\theta \otimes g$ for $X, Y$ in $\Gamma(T M)$ and $\xi \in \operatorname{RadTM}$,

$$
\begin{aligned}
0=\theta(X) g(\xi, Y) & =(\nabla g)(\xi, Y) \\
& =X \cdot g(\xi, Y)-g\left(\nabla_{X} \xi, Y\right)-g\left(\xi, \nabla_{X} Y\right) .
\end{aligned}
$$


So, $g\left(\nabla_{X} \xi, Y\right)=0, \forall X, Y \in T M$, which together with (5.1) gives $\left(\mathcal{L}_{\xi} g\right)(X, Y)=$ $\theta(\xi) g(X, Y)$ which from Proposition 3.2 means that $M$ is totally umbilical with $\alpha(\xi)=-\frac{1}{2} \theta(\xi)$.

Conversely, assume $(M, g)$ is a totally umbilical lightlike hypersurface. The 1form $\alpha$ involved in Definition 3.1 is a section of $\left(T M^{\perp}\right)^{\star}$. Then, the latter is canonically isomorphic to $\left(\left.T \bar{M}\right|_{M}\right) / T M$. Also, the projection

$$
\left.T \bar{M}\right|_{M} \longrightarrow\left(\left.T \bar{M}\right|_{M}\right) / T M
$$

has contractile fibres. Then, there exists a section $\zeta$ of $\left.T \bar{M}\right|_{M}$ such that

$$
\forall \xi \in T M^{\perp}, \quad \bar{g}(\zeta, \xi)=\alpha(\xi) .
$$

Observe that such two sections $\zeta$ differ by exactly one section of $\Gamma(T M)$. Now, let $\theta$ be the smooth 1-form on $M$ defined by

$$
\theta(X)=-2 \bar{g}(\zeta, X)
$$

and define $\nabla^{\theta}$ as

$$
\nabla_{X}^{\theta} Y=\bar{D}_{X} Y-\frac{1}{2} \theta(Y) X-\frac{1}{2} \theta(X) Y-g(X, Y) \zeta
$$

for all tangent vector fields $X$ and $Y$ in $M$ and let $\bar{D}$ be the Levi-Civita connection on ambient space $(\bar{M}, \bar{g})$.

We first show that for $X$ and $Y$ in $T M, \nabla_{X}^{\theta} Y \in T M$. Indeed,

$$
\begin{aligned}
\bar{g}\left(\nabla_{X}^{\theta} Y, \xi\right) & =\bar{g}\left(\bar{D}_{X} Y, \xi\right)-g(X, Y) \bar{g}(\zeta, \xi) \\
& \stackrel{3.3}{=} \alpha(\xi) g(X, Y)-\alpha(\xi) g(X, Y)=0 .
\end{aligned}
$$

Thus, $\nabla_{X}^{\theta} Y \in T M$ for $X$ and $Y$ tangent vector fields in $M$. Also, $\nabla^{\theta}$ is clearly a torsion-free connection on $M$. Finally, we show that $\nabla^{\theta}$ is conformal. Let $X, Y$, and $Z$ be tangent vector fields to $M$. We have,

$$
\begin{aligned}
X \cdot g(Y, Z)-g\left(\nabla_{X}^{\theta} Y, Z\right)-g\left(Y, \nabla_{X}^{\theta} Z\right)= & \bar{g}\left(\bar{D}_{X} Y, Z\right)+\bar{g}\left(Y, \bar{D}_{X} Z\right) \\
& -\bar{g}\left(\bar{D}_{X} Y, Z\right)+\frac{1}{2} \theta(Y) \bar{g}(X, Z) \\
& +\frac{1}{2} \theta(X) \bar{g}(Y, Z)+\bar{g}(X, Y) \bar{g}(\zeta, Z) \\
& -\bar{g}\left(Y, \bar{D}_{X} Z\right)+\frac{1}{2} \theta(Z) \bar{g}(Y, X) \\
& +\frac{1}{2} \theta(X) \bar{g}(Y, Z)+\bar{g}(X, Z) \bar{g}(Y, \zeta),
\end{aligned}
$$

that is,

$$
\begin{aligned}
\left(\nabla_{X}^{\theta} g\right)(Y, Z)= & \theta(X) g(Y, Z)+\left[\frac{1}{2} \theta(Z) \bar{g}(Y, X)+\bar{g}(X, Y) \bar{g}(\zeta, Z)\right] \\
& +\left[\frac{1}{2} \theta(Y) \bar{g}(Z, X)+\bar{g}(X, Z) \bar{g}(\zeta, Y)\right] .
\end{aligned}
$$

By (5.2), the terms in the last two brackets are zero. Hence, $\left(\nabla_{X}^{\theta} g\right)(Y, Z)=$ $\theta(X) g(Y, Z)$ for all tangent vector fields $X, Y$ and $Z$ in $M$ and the proof is complete. 
Remark 5.2. Observe that,

(a) if the section $\left.\zeta \in T \bar{M}\right|_{M}$ is tangent to $M$, we have $\alpha(\xi)$ identically zero and we fall in the totally geodesic case. Otherwise,

(b) $\zeta$ is nowhere tangent to $M$ and consequently, $\alpha(\xi)$ is everywhere nonzero and $(M, g)$ is proper totally umbilical. In this case, $S=\operatorname{Ker} \theta$ corresponds to the choice of a screen distribution on $M$. For such a screen distribution to be integrable, it is necessary and sufficient that $\theta$ be closed.

The above fact gives a good picture of the set of totally umbilical pairs $(M, g)$. Let us turn the problem around and relate it to one of the invariants of a given connection on $M$, the holonomy groups [7, 8. More precisely, let $M$ be a connected $(n+1)$-manifold, and $\nabla$ a torsion-free connection on $M$. We ask about necessary and sufficient conditions on $\mathrm{Hol}^{0}(\nabla)$ so that $\nabla$ is a Weyl connection of a 1-degenerate metric $g$ on $M$.

\section{HolONOMY GROUPS AND 1-DEGENERATE METRICS}

In the classical case of a manifold with a (nondegenerate) quadratic form $g$ and Weyl connection $\nabla$ with $\nabla g=\theta \otimes g$, the holonomy group of $\nabla$ at point $x$ is included in the conformal group $C O\left(g_{x}\right)=\mathbb{R}^{\star} \cdot O\left(g_{x}\right)$.

Now, let $(M, g)$ be an $(n+1)$-manifold $M$ with an everywhere co-rank one metric $g$, and $F$ the frame bundle of $M$. Then, each point of $F$ is $\left(x, e_{0}, \ldots, e_{n}\right)$, where $\left(e_{0}, \ldots, e_{n}\right)$ is a basis of $T_{x} M$, with $e_{0}$ generating the radical distribution $\operatorname{RadTM}$. Now, define $P$ to be the subset of $F$ for which $\left(e_{0}, \ldots, e_{n}\right)$ is a quasi-orthonormal basis with respect to the 1-degenerate metric $g$ with $\operatorname{sgn}(g)=(0, p, n-p)$. Then, $P$ is a $G$-structure on $M$ with fibre

$$
G=\operatorname{Aut}(T M, g)=\left\{\left(\begin{array}{cc}
a & { }^{t} B \\
0 & \\
\vdots & D \\
0 &
\end{array}\right), \quad a \in \mathbb{R}^{\star}, B \in \mathbb{R}^{n}, D \in O(p, n-p)\right\}
$$

with Lie algebra

$$
\mathcal{G}=\mathbb{R}^{n} \rtimes(\mathbb{R} \oplus s o(p, n-p)) .
$$

Now let $(M, \nabla)$ be a connected $(n+1)$-manifold $M$ with a torsion-free connection $\nabla$. We are interested in finding necessary and sufficient conditions on the holonomy group $\operatorname{Hol}(\nabla)$ for $\nabla$ to be a Weyl connection of a conformal class $[g]$ with $g$ a 1-degenerate metric on $M$.

Suppose that there exists on $M$ a 1-degenerate metric $g$ and a 1-form $\theta \in$ $C^{\infty}\left(T^{\star} M\right)$ such that $\nabla g=\theta \otimes g$. We first show that the kernel of $g$ that is the radical distribution is $\nabla$-parallel. For $\xi$ in $\operatorname{RadTM}, X$ and $Y$ in $T M$, we have

$$
\begin{aligned}
g\left(\nabla_{X} \xi, Y\right) & =X \cdot g(\xi, Y)-g\left(\xi, \nabla_{X} Y\right)-\left(\nabla_{X} g\right)(\xi, Y) \\
& =-\theta(X) g(\xi, Y)=0 .
\end{aligned}
$$

Then, $\operatorname{RadTM}$ is $\nabla$-parallel and it follows a similar argument included in the beginning of this section that $\operatorname{Hol}(\nabla)$ is a subgroup of $\mathbb{R}^{n} \rtimes \mathbb{R}^{\star} \times\left(\mathbb{R}^{\star} \cdot S O(n)\right)$; that 
is, any element of $\operatorname{Hol}(\nabla)$ has the form

$$
\left(\begin{array}{c|c}
\alpha & { }^{t} B \\
\hline 0 & \\
\vdots & \lambda C \\
0 &
\end{array}\right)
$$

with $\alpha \in \mathbb{R}^{\star}, B \in \mathbb{R}^{n}, \lambda \in \mathbb{R}^{\star}, C \in O(p, n-p)$.

Conversely, let $\nabla$ be a connection on $M$, and assume that elements of $\operatorname{Hol}(\nabla)$ have the form in (6.3). Then, there exists a rank-one subbundle of $T M$, say $L$, that is $\nabla$-parallel. Let

$$
\pi: T M \longrightarrow T M / L
$$

denote the natural projection. From (6.3), the connection $\nabla$ induces on the quotient $T M / L$ a connection $\bar{\nabla}$ explicitly defined by

$$
\bar{\nabla}_{X} \bar{Y}=\pi\left(\nabla_{X} Y\right)
$$

for all tangent vectors $X, Y$ in $T M$, with $\bar{Y}=\pi(Y)$. Relation (6.4) is well defined since for $Y^{\prime}=Y+U$ with $U \in L$, we have

$$
\begin{aligned}
\bar{\nabla}_{X} \overline{Y^{\prime}} & =\pi\left(\nabla_{X} Y^{\prime}\right)=\pi\left(\nabla_{X} Y+\nabla_{X} U\right) \\
& =\pi\left(\nabla_{X} Y\right)+\pi\left(\nabla_{X} U\right)=\pi\left(\nabla_{X} Y\right)=\bar{\nabla}_{X} \bar{Y},
\end{aligned}
$$

where we used the fact that $L$ is $\nabla$-parallel. Thus, (6.4) is independent of the choice of $Y$ in $\bar{Y}$.

On the other hand, it follows from (6.3) and (6.4) that

$$
\operatorname{Hol}(\bar{\nabla}) \subset \mathbb{R}^{\star} \cdot O(p, n-p) .
$$

Then, from (6.5), $\bar{\nabla}$ is a connection on the vector bundle $T M / L$ over $M$, that is, compatible with a conformal class $[\bar{g}]$, where $\bar{g}$ is a nondegenerate $(0,2)$ tensor field on the vector bundle $T M / L$. Alternatively, there exists a 1 -form $\theta \in C^{\infty}\left(T^{\star} M\right)$ such that

$$
\left(\bar{\nabla}_{X} \bar{g}\right)(\bar{Y}, \bar{Z})=\theta(X) \bar{g}(\bar{Y}, \bar{Z})
$$

with $\bar{Y}=\pi(Y)$ and $\bar{Z}=\pi(Z)$.

We now define on $M$ a $(0,2)$ tensor $g$ as follows. For tangent vector fields $X, Y$ in $T M$,

$$
g(X, Y)=\bar{g}(\pi X, \pi Y) .
$$

Clearly, such a $g$ defines on $M$ a degenerate (everywhere) co-rank one metric, with radical distribution $\operatorname{RadTM}=L$. Thus, one has for $X, Y$ and $Z$ in $T M$,

$$
\begin{aligned}
\left(\nabla_{X} g\right)(Y, Z) & =X \cdot g(Y, Z)-g\left(\nabla_{X} Y, Z\right)-g\left(Y, \nabla_{X} Z\right) \\
& =X \cdot \bar{g}(\pi Y, \pi Z)-\bar{g}\left(\pi\left(\nabla_{X} Y\right), \pi Z\right)-\bar{g}\left(\pi Y, \pi\left(\nabla_{X} Z\right)\right) \\
& =X \cdot \bar{g}(\pi Y, \pi Z)-\bar{g}\left(\bar{\nabla}_{X} \bar{Y}, \pi Z\right)-\bar{g}\left(\pi Y, \bar{\nabla}_{X} \bar{Z}\right) \\
& =\left(\bar{\nabla}_{X} \bar{g}\right)(\bar{Y}, \bar{Z})=\theta(X) \bar{g}(\bar{Y}, \bar{Z}) \\
& =\theta(X) g(Y, Z) .
\end{aligned}
$$

Thus, $\nabla$ is a Weyl connection for the conformal class $[g]$ of the 1-degenerate metric $g$ on $M$ given by (6.7). Thus, we have proved the following. 
Theorem 6.1. Let $M$ be a connected $(n+1)$-manifold, and let $\nabla$ be a torsionfree connection on TM. Then $\nabla$ is a Weyl connection of a 1 -degenerate metric $g$ on $M$ if and only if $\mathrm{Hol}(\nabla)$ is, up to conjugation in $G l(n+1, \mathbb{R})$, a subgroup of $\mathbb{R}^{n} \rtimes\left[\mathbb{R}^{\star} \times\left(\mathbb{R}^{\star} \cdot O(p, n-p)\right)\right]$.

Remark 6.2. Observe that in Theorem 6.1, one can consider instead the restricted holonomy group $\operatorname{Hol}^{0}(\nabla)$. Then, $\nabla$ is a Weyl connection of a 1-degenerate metric $g$ on $M$ if and only if

$$
\mathrm{Hol}^{0}(\nabla) \subset \mathbb{R}^{n} \rtimes\left[\mathbb{R}_{+}^{\star} \times\left(\mathbb{R}_{+}^{\star} \cdot S O_{0}(p, n-p)\right)\right],
$$

where $S O_{0}(p, n-p)$ is the connected component of Identity in $S O(p, n-p)$. Recall that if $M$ is 1-connected, then $\operatorname{Hol}(\nabla)=\operatorname{Hol}^{0}(\nabla)$.

\section{REFERENCES}

1. M.A. Akivis and V.V. Goldberg Lightlike hypersurfaces on manifolds endowed with a conformal structure of Lorentzian signature, Acta Appl. Math. 57 (1999), 255-285. MR1722041 (2001b:53090)

2. C. Atindogbe, J.-P. Ezin, and J. Tossa, Pseudo-inversion of degenerate metrics, International Journal of Mathematics and Mathematical Sciences 55 (2003), 3479-3501. MR.2019290 (2005e:53107)

3. C. Atindogbe, J.P. Ezin, J. Tossa, Reduction of the co dimension of Lightlike Isotropic Submanifolds, Journal of Geom. and Phys 42 (1-2) (2002), 1-11. MR.1894071 (2003b:53076)

4. C. Atindogbe, K.L. Duggal, Conformal screen on lightlike hypersurfaces, International Journal of Pure and Applied Mathematics, vol. 11, 4, 2004, 421-442. MR2039644 (2004k:53102)

5. A. Bejancu and K.L. Duggal, Lightlike submanifolds of semi-Riemannian Manifolds and Applications, Mathematics and Its Applications, 364 (1990). MR1383318 (97e:53121)

6. L. Berard-Bergery and A. Ikemakhen, On the holonomy of Lorentzian manifolds, Differential geometry: geometry in mathematical physics and related topics, Proc. Sympos. Pure Math 54 (1993), 27-40. MR1216527 (94d:53106)

7. A.L. Besse Einstein manifolds, Springer, Berlin, 1987. MR0867684 (88f:53087)

8. D.D. Joyce, Compact manifolds with special holonomy, Oxford Science Publications, 2000. MR.1787733 (2001k:53093)

9. S. Ivanov, Einstein-Weyl structures on compact conformal manifolds, Quarterly Journal of Mathematics. 50 (1999), 457-462. MR.1726786 (2001d:53049)

10. S.W. Hawking and G.F.R. Ellis, The Large Scale Structure of Spacetime, Cambridge University Press, Cambridge, (1973). MR0424186 (54:12154)

11. D.N. Kupeli, On null submanifolds in spacetimes, Geom. Dedicata 23 (1987), 33-51. MR0886772 (88j:53068)

12. B. O'Neill, Semi-Riemannian geometry with Applications to Relativity, Academic Press, New York, (1983). MR0719023 (85f:53002)

13. A. Zeghib, Remarks on Lorentzian symmetric spaces, Compositio Math. 140 (2004), 16751678. MR2098408 (2005g:53127)

14. A. Zeghib, Isometry groups and geodesic foliations of Lorentz manifolds. Part II: Geometry of analytic Lorentz manifolds with large isometry groups, GAFA, Geom. funct. anal. 9 (1999), 823-854. MR:1719610 (2001g:53060)

Institut De Mathématiques et de Sciences Physiques, 01 BP 613, Porto-Novo, Bénin E-mail address: atincyr@imsp-uac.org

Institut Elie Cartan, Université Henri Poincaré, Nancy I, B.P. 23954506 VandeuvreLÈs NANCY Cedex, France

E-mail address: berard@iecn.u-nancy.fr 